\title{
Silica
}

\section{CANCER MORBIDITY AND QUARTZ EXPOSURE IN SWEDISH IRON FOUNDRIES}

Håkan Westberg, Lena Andersson, Carl-Göran Ohlson, Ing-Lisss Bryngelsson, Yen Ngo Örebro University Hospital, Örebro, Sweden

10.1136/oemed-2011-100382.127

Objectives The aim of this study was to determine cancer morbidity for Swedish iron foundry workers with special reference to quartz exposure.

Methods The cohort of 3045 men employed between 1913 and 2005 and with $>1$ year of exposure was followed for cancer morbidity from 1958 to 2004. Standardised incidence ratios (SIRs) with $95 \%$ CIs were determined by comparing the observed number of incident cancers to the Swedish cancer register. Exposure measures were assessed from personal files and a quartz measurement database of 2822 air samples.

Results Overall cancer morbidity was not increased (SIR 1.00 ; $95 \%$ CI 0.90 to 1.11), however lung cancer was significantly enhanced (SIR 1.61; 95 \% CI 1.20 to 2.12). Dose response for lung cancer was determined for duration of employment, duration of exposures and cumulative quartz for latency periods $>20$ years. Significant findings for lung cancer and cumulative quartz were determined for quartz doses of $1-2 \mathrm{mg} / \mathrm{m}^{3}$ *year, SIR 2.88 (95\% CI 1.44 to 5.16 ), and for $>2 \mathrm{mg} / \mathrm{m}^{3}$ *year a SIR of 1.68 (95\% CI 1.07 to 2.52$)$. For cancer sites with at least 5 observed cases and a SIR $>1.25$, non-significant risks were determined for liver, larynx, testis, the urinary organs, muscle of the connective tissue and lymphatic leukemia.

Conclusions An increased lung cancer risk for Swedish foundry workers related to quartz exposure was determined. 